Meta

Journal des traducteurs

Translators' Journal

\title{
Le rôle des annotations de textes dans l'enseignement de la traduction
}

\section{Robert Larose}

Volume 29, numéro 2, juin 1984

URI : https://id.erudit.org/iderudit/003403ar

DOI : https://doi.org/10.7202/003403ar

Aller au sommaire du numéro

Éditeur(s)

Les Presses de l'Université de Montréal

ISSN

0026-0452 (imprimé)

1492-1421 (numérique)

Découvrir la revue

Citer cet article

Larose, R. (1984). Le rôle des annotations de textes dans l'enseignement de la traduction. Meta, 29(2), 143-151. https://doi.org/10.7202/003403ar d'utilisation que vous pouvez consulter en ligne.

https://apropos.erudit.org/fr/usagers/politique-dutilisation/ 


\section{LE RÔLE DES ANNOTATIONS DE TEXTES DANS L'ENSEIGNEMENT DE LA TRADUCTION ${ }^{*}$}

ROBERT LAROSE

Au début des années 70, l'enseignement de la traduction dans les universités canadiennes réservait peu de place, si tant est qu'elle en réservât une, à l'explication des textes à traduire telle que nous l'entendons aujourd'hui. Il s'agit à plusieurs égards d'une époque creuse dans l'histoire de l'enseignement de la traduction au Canada. C'est alors qu'avaient lieu ces fameuses séances, où les étudiants devaient maintes fois traduire à vue, sous le regard impatient du professeur, des textes qui venaient tout juste de leur être remis. Ils ne se voyaient pas seulement tenus de traduire, à la vitesse de la pensée du professeur, des textes dont on ne précisait pas l'origine, ni la destination, mais ils n'étaient, de surcroît, assistés d'aucune espèce de mise en garde écrite qui aurait pu leur éviter nombre de pertes de temps et d'énergie, de mortifications, sinon de traumatismes. Nous n'envisageons pas ici les corrections des travaux et examens, mais précisément les exercices faits en classe.

À quoi pouvait bien ressembler un cours de traduction à cette époque ? Le professeur arrivant en classe remettait aux étudiants un certain nombre de textes, non annotés, il va sans dire. Quelques-uns étaient traduits séance tenante : c'étaient les traductions à vue dont nous venons de parler. D'autres devaient être préparés comme devoirs, pour être examinés en classe la semaine suivante. C'est de ce second type d'exercice qu'il sera question ici. Dans ce cas également, aucune note n'accompagnait les textes à traduire. Aussi, probablement sans en avoir trop conscience, c'était bien souvent vers une sorte d'abattoir traductionnel, et non un cours de correction, que s'acheminaient les étudiants à la séance suivante. La salle de classe devenait le lieu d'une élimination dont le caractère erratique ne conférait certes pas à ladite élimination une rationalité de type darwinien, et n'était plus alors que théoriquement un lieu d'apprentissage. Faut-il rappeler qu'à cette époque, il n'existait en fait de livres portant sur la traduction que quelques recueils de textes, généralement à forte teneur littéraire, et donc inadaptés aux besoins d'un futur traducteur de textes pragmatiques ${ }^{1}$. Nous songeons en particulier aux Chemins de la traduction de L. Bonnerot dont les notes évoquent, tout en étant loin de présenter le même degré de précision et de pertinence, celles qui figurent à l'Appendice III de la Stylistique comparée du français et de l'anglais, de J.-P. Vinay et J. Darbelnet. Les notes de Bonnerot n'avaient pas pour objectif d'aider l'étudiant à traduire le texte

* Texte d'une conférence prononcée à l'École de traducteurs et d'interprètes de l'Université d'Ottawa en janvier 1982.

1. L'expression " texte pragmatique " est à comprendre au sens que lui donne J. Delisle, soit : « les textes qui servent essentiellement à véhiculer une information et dont l'aspect esthétique n'est pas l'aspect dominant" (p. 22). 
anglais ou français, mais à lui faire constater les différences entre les deux versions, l'une anglaise, l'autre française, qui lui étaient soumises. Pour l'un et l'autre de ces ouvrages, c'est dans une perspective comparatiste, donc dans celle d'une démarche postérieure à l'activité traduisante, que se situaient les notes d'accompagnement.

Depuis 1975, trois livres surtout on marqué à des degrés divers l'enseignement de la traduction au Canada. Le premier en date, Pratique de la traduction - Version générale de J.-P. Bénard et $\mathbf{P}$. Horguelin, paru en 1977, est un recueil de textes destiné aux étudiants des cours de version générale. Malheureusement, seuls trois des vingt-huit textes qu'il contient ont été annotés. Néanmoins, à la différence de Bonnerot et de Vinay et Darbelnet, dont les remarques pour un texte donné ne dépassent généralement pas la demi-douzaine, Bénard et Horguelin annotent à peu près toutes les unités de traduction et ne limitent pas leurs commentaires aux difficultés lexicales. Larose, Sparer et Vitale feront plus tard de même dans les deux tomes de leur Guide de la traduction appliquée, parus en 1978 et 1980 . Cependant, les annotations de Bénard et Horguelin diffèrent de celles de ce dernier ouvrage en ce qu'elles consistent à illustrer un modèle de traduction proposé dans les premières pages du recueil. Les vingt-cinq textes censés être à traduire par les étudiants, peut-être chez eux, peut-être en classe, ne sont pas accompagnés de notes d'orientation ; seuls le sont les corrigés, vendus séparément, et offerts uniquement aux professeurs.

Le troisième ouvrage est la thèse de Jean Delisle, publiée en 1980, l'Analyse du discours comme méthode de traduction. Comme l'indique ce titre, l'explication du texte de départ ou, si l'on préfêre, l'analyse du discours d'origine, joue un rôle prépondérant dans la pédagogie de la traduction aux yeux de cet auteur. $\mathbf{L}$ '" Objectif III " de Delisle, soit l'explication de texte, consiste à "découvrir le texte en le lisant méticuleusement afin de le comprendre jusque dans ses nuances les plus subtiles » (p. 141). L'explication du texte A Funny Thing Happened on My Way se présente comme un questionnaire que l'auteur divise en quatre parties qu'il intitule comme suit : «1) Les clés du texte, c'est-àdire tout ce qui le situe et en précise le cadre ; 2) les connaissances non linguistiques nécessaires à sa compréhension ; 3) le dépistage des sous-entendus et des allusions ; 4) l'interprétation sémantique et stylistique de certains mots, syntagmes ou idiotismes " (p. 142). Ces questions pourraient très bien prendre la forme de notes qui accompagneraient tout texte remis à des étudiants pour qu'ils le traduisent ultérieurement. L'auteur ne dit-il pas, à la page 144 , que " tout passage présentant un intérêt du point de vue de la traduction peut faire l'objet d'une note explicative ou justificative "?

Pour qu'on puisse mieux juger de l'importance des notes et apprécier leurs avantages et inconvénients, il nous semble opportun d'examiner un exemple de texte annoté, en l'occurrence une description d'origine canadienne des fonctions du vérificateur externe, qui a été utilisé dans un cours de traduction de deuxième année intitulé "Textes de comptabilité ». Précisons qu'en raison de sa relative simplicité, ce texte aurait pu également être proposé dans un cours de version générale de première année.

En ce qui concerne le moment où ces notes sont à utiliser, il est essentiel de préciser que l'étudiant ne devrait s'y reporter qu'après avoir produit un travail préliminaire, spontané, ou premier jet. Il évitera ainsi le risque de présenter une traduction élaborée "à ras de texte " parce que faite à coup de notes, et donc non perçue dans son homogénéité. De plus, il est toujours rappelé aux étudiants que les notes qui accompagnent un texte ne sont pas censées fournir les seules et uniques façons de traduire.

Notre but n'est pas d'illustrer une méthode de traduction, mais d'envisager la façon dont on peut appliquer concrètement une règle fondamentale en pédagogie de la traduction, c'est-à-dire celle consistant à estimer qu'il vaut mieux prévenir les fautes que les corriger. 


\section{EXEMPLE D'ANNOTATIONS DE TEXTE}

\section{Role of the External Auditor}

The objective of an audit of financial statements is to express an opinion on the fairness with which they present the financial position, results of operations and changes in $f$ nancial position in accordance with generally accepted accounting principles, or in special circumstances another appropriate disclosed basis of accounting, consistently applied. It is not the objective of an audit to express an opinion as to the future viability of an organization nor the efficiency or effectiveness with which its affairs have been conducted.

The operations ${ }^{1}$ of an organization ${ }^{2}$ are under the control ${ }^{3}$ of management $t^{4}$, which has the responsibility for the accurate recording ${ }^{5}$ of transactions ${ }^{6}$ and the preparation ${ }^{7}$ of financial statements 8 in accordance with generally accepted accounting principles 9 . These responsibilities include designing and maintaining 10 accounting records ${ }^{11}$ and internal controls $^{12}$, selecting and applying accounting policies ${ }^{13}$, safeguarding assets and preventing and detecting fraud and 14 error. An audit 15 of the financial statements does not relieve management of its responsibilities. The auditor 16 may make suggestions as to the form or content of the financial statements or he may draft them in whole or in part, based on management's accounting records. However, financial statements remain the representations $^{17}$ and responsibility of management 18 .

In the performance of an audit of financial statements, the auditor complies with generally accepted auditing standard, which relate to the auditor's qualifications, the performance of his examination and the preparation of his report.

Mise en situation ${ }^{2}$

Le texte à traduire est tiré d'une monographie de l'Institut canadien des comptables agréés, intitulée The Management Report (Le rapport de la direction) et publiée en 1981. Cette étude s'adresse à des experts-comptables canadiens qui doivent réfléchir sur la nécessité de joindre un rapport de la direction aux états financiers de l'entreprise. Elle tire son origine des poursuites en matière de responsabilité des états financiers dont été victimes de nombreux experts-comptables américains.

Principaux documents et ouvrages de référence ${ }^{3}$

DUBUC, Robert (1974) : Vocabulaire de gestion, Montréal, Leméac.

PYLE, WHITE, LARSON, ZIN et COLETTE (1980) : Initiation à la comptabilité financière et administrative, Georgetown (Ont.), Irwin-Dorsey.

SYLVAIN, Fernand (1977) : Dictionnaire de la comptabilité, Toronto, I.C.C.A.

"The Adams Report", C.A. Magazine, avril 1978. (Comparaison des versions anglaise et française du rapport Adams.)

Manuel de l'I.C.C.A., Toronto, I.C.C.A.

Terminologie comptable, Ordre des comptables agréés du Québec.

Remarques préliminaires relativement à la typologie des notes

L'absence de notes après une forme linguistique signifie en principe qu'elle peut être traduite plus ou moins littéralement. L'annotateur s'intéresse donc surtout aux cas " obliques ", et son but est en définitive de mettre en lumière toute littéralité abusive susceptible d'apparaître dans les copies d'étudiants. Les notes d'orientation peuvent être

2. La mise en situation, ou macro-structure textuelle, correspond pour Delisle à la première étape d'explication du texte ("Les clés du texte").

3. Liste délibérément limitée à quelques titres. Une bibliographie trop longue, pour un texte donné, ne peut que désemparer l'étudiant, ou le mener à accumuler une masse de renseignements dont il risquera de ne pas pouvoir dégager l'essentiel. 
classées en trois catégories principales : elles peuvent être encyclopédiques, linguistiques ou traductionnelles.

a) Notes encyclopédiques : Destinées à permettre à l'étudiant de se renseigner sur les choses, les concepts, à lui permettre de comprendre avant de traduire. Il est recommandé de ne pas fournir de solutions dans les notes, mais d'orienter l'élève-traducteur vers des sources documentaires aisément accessibles (ouvrages disponibles dans les bibliothèques, publications d'entreprises, périodiques spécialisés, etc.).

b) Notes linguistiques : Destinées à permettre à l'étudiant de mieux interpréter le texte de départ et de mieux rédiger en langue d'arrivée. Ces notes procèdent de la détermination préalable, à l'intérieur du texte de départ, des plus petits segments susceptibles d'entraîner une faute de traduction, et du découpage subséquent de ce texte.

En fait, toute typologie des annotations de textes en traduction repose sur une typologie des fautes qu'un élève-traducteur de niveau moyen risque de commettre. On ne le mettra en garde contre un anglicisme donné que s'il y a une forte probabilité d'apparition. Il n'est pas inutile de mentionner que le découpage en unités de traduction, tel qu'il est effectué par Vinay et Darbelnet, diffêre de celui dont il est question ici. Chez ces auteurs, la segmentation proposée de même que leurs annotations ${ }^{4}$, visent à permettre de comparer les caractéristiques propres à chaque langue et ce sont des activités postérieures à celle de la traduction qu'ils ont faite eux-mêmes. Au contraire, la segmentation et les annotations de textes en traduction, telles que nous les concevons, remplissent un double objectif d'exégèse textuelle et de prévention de fautes.

c) Notes traductionnelles : Destinées à permettre à l'étudiant d'appliquer les techniques courantes de passage d'une langue à une autre (transpositions, modulations, etc.).

\section{Explication des notes}

Il ne faut attribuer qu'une valeur d'exemple aux explications qui accompagnent les notes suivantes : elles pourraient être nettement plus sommaires ou plus développées. Nous recommandons fortement que de telles explications accompagnent les corrigés, écrits ou oraux, présentés aux étudiants lorsque leurs devoirs leur sont rendus. De cette façon, à chaque note correspondra à la fois un commentaire qui l'explicitera à toutes fins utiles, et bien entendu, l'une des solutions acceptables pouvant permettre aux étudiants de saisir la différence de formulation qu'exige le passage de l'anglais au français, pour le cas où celle-ci leur aurait échappé. À cet égard, nous ne voyons pas pourquoi le professeur éviterait de remettre sa propre version de la traduction, ou toute version établie par quelqu'un d'autre, à moins de croire qu'il voudrait, d'une année à l'autre, utiliser les mêmes textes jusqu'à la fin de sa carrière, tout en prétendant empêcher que se crée un " marché noir » des corrigés. Soulignons à ce sujet combien il serait injustifié, pour écarter la remise aux étudiants d'un corrigé, d'invoquer l'inutilité de ce dernier, ou de lui prêter des dangers. Contrairement à ce que certains préjugés mènent parfois à affirmer, la grande majorité des étudiants lisent attentivement les corrigés, et bien peu d'entre eux sont assez naifs pour croire que la seule traduction possible et correcte est toujours celle du professeur.

4. Ce sont celles contenues à l'Appendice III de la Stylistique comparée du français et de l'anglais de J.-P. Vinay et J. Darbelnet. 
1. operations : a) que signifie ce mot ? b) en quoi se distingue-t-il de transactions (note 6) ? c) distinguez "opérations" et "exploitation". (V. R. Dubuc, Vocabulaire de gestion; G. Dagenais, Dictionnaire des difficultés de la langue française au Canada, et Terminologie comptable, $\mathrm{n}^{\circ}$ 10.)

Operations, qui appartient au vocabulaire anglais de la gestion, signifie l'ensemble des activités qui assurent le fonctionnement d'une entreprise et se rend en français par "exploitation" (v. Dubuc). "En termes de commerce et de finance, son similigraphe français veut dire "transaction, affaire " : opération de banque, opération au comptant, opération forfaitaire. " (Dagenais)

Transactions employé au sens d'affair, de piece of business, désigne un acte accompli entre des individus, soit dans le commerce, soit dans la vie ordinaire. Comme le soulignent Funk \& Wagnalls, a transaction is a single action (Terminologie comptable, $\mathrm{n}^{\circ}$ $10)$.

Dans le présent contexte, operations se rend en français par "exploitation ", et transactions par "opérations".

2. organization : s'agit-il de " l'agencement rationnel des unités de travail au sein de l'entreprise " ou de "la structure administrative d'une entreprise »? (V. Dubuc)

Il ne s'agit ici que de la structure administrative évoquée dans la note, donc, de son organisation (v. Dubuc). Mais dans le présent contexte, ce dernier mot a pour synonyme le terme "entreprise", qu'on emploiera de préférence pour éviter un mot de plus en -tion dans la phrase française.

3. under the control : l'expression "sous le contrôle de " est-elle sémantiquement acceptable ici ? Dans la négative, faire ressortir le concept d'« autorité ». (V. Control dans P. Daviault, Langage et traduction, et dans G. Dagenais.)

La locution "sous le contrôle de" existe, mais seulement pour parler de surveillance ou de supervision. To control veut dire " exercer une influence dominante sur, diriger, dominer, gouverner, maîtriser ». Ce sont des verbes comme diriger, dominer, mấtriser et régir et des substantifs comme direction, domination, autorité et maîtrise qu'il faut employer pour traduire les idées véhiculées par les mots anglais control et to control (v. Dagenais). Ici, il y a lieu, soit de faire une transposition verbale ("relever de"), soit d'employer une locution du type "soumettre à l'autorité de".

4. management : a) l'emprunt " management "a-t-il reçu droit de cité en français ? (V. dictionnaires usuels de la langue.) b) dans l'affirmative, en quoi se distingue-t-il d'autres mots appartenant au même champ lexical ? (V. Dubuc)

Dans l'un des arrêtés du gouvernement français en matière de terminologie (Journal officiel, 18 janvier 1973), non seulement l'emprunt " management " a été approuvé, mais son usage a été présenté comme obligatoire dans les textes officiels. L'Office de la langue française du Québec n'a pas repris cette recommandation et a endossé les équivalents proposés dans le Vocabulaire de gestion de R. Dubuc, à savoir : a) organisation (philosophie de l'activité créatrice qui dégage de l'expérience les règles de l'utilisation la plus efficace du travail et des capitaux mise en oeuvre), $b$ ) gestion (ensemble des activités nécessaires pour atteindre les objectifs de l'entreprise), c) direction (ensęmble de ceux qui dirigent une entreprise) et $d$ ) cadres (ensemble du personnel exerçant une autorité à divers palliers de l'entreprise). Il s'agit donc dans ce cas-ci de la solution c).

5. for ... recording : a) for : éviter la lourdeur d'une structure commençant par "pour "; b) recording : infinitif ou substantif? 
Enregistrer et enregistrement employés pour parler d'inscription sur un registre ne se disent qu'en parlant des choses (v. Dagenais). L'infinitif permettrait d'alléger la phrase et d'éviter une cascade de substantifs, ou "substantivite».

6. transactions : voir note 1.

7. preparation : verbe ou substantif selon la solution qui a été retenue au no $5 b$. bilité.

8. financial statements : voir la définition de F. Sylvain, Dictionnaire de la compta-

Selon Sylvain, il s'agit d'un rapport renfermant plusieurs rapports financiers.

Financial statement : bilan, état des résultats, état des bénéfices non répartis, état des recettes et déboursés, état de l'évolution de la situation financière ou tout autre rapport financier présenté en bonne et due forme. Équivalent français : état financier.

À noter que le synonyme québécois d'état financier, "rapport financier ", est inusité en France.

9. in ... principles : a) voir l'expression consacrée dans le Manuel de l'I.C.C.A. b) generally accepted accounting principles : v. Pyle, Larson, Zin et Colette, Initiation à la comptabilité financière et administrative, p. 14.

a) L'expression correspondante fournie par le Manuel de l'I.C.C.A. est "conformément aux principes comptables généralement reconnus ». b) D'après Pyle et al., ces principes sont :

1. Le principe du coût

2. Le principe d'objectivité

3. Le principe de la réalisation du revenu

4. Le principe du rapprochement

5. I a présentation fidèle et complète des états financiers

6. L'importance des postes et des montants

7. Le principe de continuité

8. L'attitude prudente

10. maintaining : a) "maintenir » ou «tenir »? b) l'équivalent choisi est-il cooccurrent, c'est-à-dire compatible à la fois avec records et internal controls?

Rapprocher maintain de controls en l'absence d'un contexte de durée semble abusif. On " applique" des internal controls (tout comme des accounting policies), on n'en assure certainement pas la «tenue»!

11. accounting records : voir la définition de Sylvain.

Accounting records : les livres comptables ainsi que les pièces justificatives et toute autre documentation probante. Équivalent français : registres (comptables), livres (comptables). V. Sylvain.

12. internal controls : voir Sylvain et le bulletin $n^{\circ} 26$ de Terminologie comptable.

Internal control : organisation structurelle de l'entreprise définie par sa haute direction et agencement des systèmes établis en vue d'assurer, dans toute la mesure du possible, la gestion saine et efficace des affaires, la conservation du patrimoine ("Conservation du patrimoine » est un équivalent admissible de safeguarding assets), la fiabilité des registres comptables et la publication en temps opportun des informations financières. Équivalent français : contrôle interne (v. Sylvain). 
Dans le bulletin $n^{\circ} 26$ de Terminologie comptable, le contrôle interne est défini comme un "ensemble de systèmes comportant des éléments " (méthodes, procédés, organisation des activités) dont chacun est également appelé "contrôle interne".

13. accounting policies : $a$ ) v. Sylvain, $b$ ) le terme " politique " relève-t-il davantage de la gestion que de la comptabilité ? (v. Dubuc et le bulletin no 16 de Terminologie comptable), c) "politique " prend-il d'habitude la marque du pluriel comme en anglais ?

a) L'expression accounting policies a un sens collectif : elle est synonyme de accounting conventions, locution désignant aussi bien les postulats et les principes que les procédés comptables. Équivalent français : conventions comptables (v. Sylvain). Dans leur Initiation à la comptabilité financière et administrative (p. 13), Pyle et al. énumèrent quatre conventions généralement reconnues :

1. La personnalité de l'entreprise

2. La permanence de l'entreprise

3. La comptabilisation des opérations en monnaie

4. La périodicité de la présentation des états

b) «politique " pour rendre policy (sing.) appartient au vocabulaire de la gestion.

c) Réponse négative. Le nombre en français du Québec, est un domaine grammatical négligé, en ce sens qu'il est courant ici d'ajouter un $s$ à des mots qui ne se prêtent pas à un dénombrement. "Politique " est l'un d'entre eux. On peut également citer argents, coûts, actifs, stratégies, tactiques, problématiques, etc.

14. and : éviter la lourdeur de style qui consisterait à rendre littéralement en français les trois " and".

15. audit : a) v. la définition de Sylvain, $b$ ) y a-t-il une distinction à faire entre " vérification ", " audit ", " révision " et " contrôle " entre le Canada et la France ? (V. bulletin $\mathrm{n}^{\circ} 19$ de Terminologie comptable.)

audit : étude critique des registres et preuves comptables, dans le but de déterminer si les états financiers de l'entreprise reflètent fidèlement la situation financière de celle-ci à une date donnée et les résultats de son exploitation pour l'exercice clos à cette date, conformément aux principes comptables généralement reconnus (v. Sylvain).

$\mathrm{Au}$ Québec, l'équivalent français employé est "vérification ». Par ailleurs, la personne exerçant ces fonctions est appelée "réviseur" en Europe, et la fonction ellemême, "révisorat " en Belgique, et " révision des comptes" en France. Ces différents termes présentent l'inconvénient de faire supposer que ce qui a été fait est nécessairement à modifier, et au Québec, l'usage semble justifié de continuer à employer " vérificateur». On remarquera enfin que l'anglicisme sémantique "auditeur»se rencontre de plus en plus fréquemment en Europe, à la place des termes "vérificateur" et "réviseur».

En ce qui concerne les mots " contrôle " et " révision ", ils sont synonymes. Toutefois, leur emploi varie selon le contexte : "contrôle" est généralement utilisé par le "commissaire aux comptes" (mandataire des actionnaires), et " révision ", par l'expertcomptable (généralement écrit sans trait d'union en Europe), qui est le mandataire des dirigeants de l'entreprise ou de tiers intéressés. (Terminologie comptable, bulletin n ${ }^{\circ} 19$. .)

16. auditor : "réviseur ", "vérificateur » ou «auditeur "?

Voir note 15.

17. representations : faux ami (v. Daviault). 
L'anglais representation désigne un simple exposé des faits. En français ce mot signifie " remontrance ", "observation ayant le caractère d'un blâme mesuré " (Lexis).

18. responsibility of management : mots à traduire en les rattachant respectivement à deux autres termes : $a$ ) of management : à rattacher à representations, $b$ ) responsibility : à rattacher à management à l'aide d'une relative which is responsible for them.

La périphrase anglaise which is responsible for them permet souvent une formulation plus fluide. La traduction proposée sera donc : "Il reste que les états financiers constituent des exposés descriptifs de la direction, qui est responsable de leur établissement. "

\section{Version française}

L'exploitation d'une entreprise relève de la direction qui a la responsabilité d'enregistrer exactement les diverses opérations et de préparer les états financiers, conformément aux principes comptables généralement reconnus. Ces responsabilités comprennent l'élaboration et l'application des contrôles internes, de même que la tenue des registres comptables, la sélection et l'application des conventions comptables, la protection des biens, la prévention et la détection des fraudes ou erreurs. La vérification des états financiers ne dégage toutefois pas la direction de ses responsabilités. Le vérificateur peut faire des suggestions relatives à la forme ou au contenu des états financiers, ou il peut se charger de leur rédaction partielle ou totale, faite à partir des registres comptables de la direction. Il reste que les états financiers constituent des exposés descriptifs de la direction, qui est responsable de leur établissement.

Inconvénients possibles des notes

Il va sans dire que la rédaction d'une note d'orientation peut être plus ou moins heureuse, et que toutes ne sont pas nécessairement d'une clarté lumineuse. Il n'est pas toujours facile, en effet, de traduire exactement l'intention d'une note. Et même quand on pense y être parvenu, l'étudiant qui interprète mal une note et perd des points a tendance à prétendre que son erreur est imputable à la "mauvaise rédaction " de la note, c'est-à-dire en général à son ambiguîté réelle ou supposée. D'autre part, le risque existe que l'étudiant considère une note, non comme une suggestion, mais comme une obligation. Ces difficultés, inhérentes à ce genre de texte où il faut se garder d'en dire trop, ne sauraient être en aucun cas des raisons d'en écarter le principe.

Deux autres risques, qui n'infirment pas plus le principe énoncé ci-dessus, méritent pourtant une attention particulière. Le premier est celui qui résulterait d'un nombre excessif de notes accompagnant un texte. Comme les annotations varient selon le niveau des étudiants et les objectifs du professeur (s'adressent-elles surtout aux plus forts, aux plus faibles, à l'étudiant moyen ? ont-elles pour but d'inciter l'étudiant à des recherches terminologiques ou autres? etc.), l'annotateur doit tenir compte de la variété de ces objectifs lorsqu'il détermine la nature et le nombre. Toutefois, la recommandation générale suivante adressée par Bénard et Horguelin aux professeurs qui utilisent leur Pratique de la traduction nous semble tout à fait pertinente : " $\overline{\mathrm{A}}$ mesure que le cours progresse, le professeur réduit le nombre de mises en garde, pour finalement laisser les étudiants voler de leurs propres ailes et donner leur pleine mesure dans les textes qui font appel à tout leur bagage linguistique et culturel. " (Recueil des corrigés, p. 3) Le deuxième risque est une mauvaise détermination, par l'étudiant, du moment où il devrait se reporter aux notes. Ces deux risques ont donné lieu, de la part d'un certain nombre d'étudiants de l'université de Montréal qui ont utilisé le tome 2 du Guide de la traduction appliquée, d'observations dont Daniel Slote a relaté la teneur dans Meta (26-2). 
"The notes that follow the texts are thought-provoking, meticulously prepared and useful to the beginning student. My only criticism is their plentifulness that can sometimes confuse the more mature student. As I have pointed out, however, to my students in the qualifying year of the Masters Programme at the Université de Montréal the better student should read the notes after the work is done. Certain revisions can be performed at that time but not before nor during the translation process. The beginning student on the other hand should study the notes very carefully before any work is done on the text itself. These remarks are based on actual reactions that were transmitted to me by those students who experimented with the Guide in my course on General Translation this year. »

\section{Avantages des notes}

Si nous avons voulu un moment nous faire l'avocat du diable, il ne faudrait quand même pas croire que les quelques risques afférents aux annotations de texte approchent, même de loin, les avantages qu'ils présentent, comme le démontre toute l'expérience que nous en avons acquise. L'étudiant de version générale laissé à lui-même devant un texte éprouve souvent des difficultés et sensations douloureuses analogues à celles que nous avons eu le déplaisir de connaître pendant nos études de traduction.

Sur le plan méthodologique, l'étudiant risque moins de se perdre dans des recherches aussi dispersées qu'infructueuses. Surtout, ces recherches peuvent être mieux dirigées, grâce en particulier aux références qui se trouvent à la fin des notes, qui doivent éviter des pertes de temps et d'énergie. En ce qui concerne les techniques de traduction elles-mêmes, c'est-à-dire le recours constant et conscient aux transpositions, modulations et autres mécanismes désormais classiques du passage d'une langue à une autre, ces techniques finissent par devenir des automatismes chez l'étudiant, et les indications données à ce sujet peuvent l'aider à se libérer plus facilement de la tyrannie de l'original. L'utilisation des notes dans tous les cours, hormis peut-être dans certains de troisième année, est aussi une façon de rendre plus homogène l'enseignement de la traduction (et peut-être même aussi celui de la terminologie et de la documentation) et, certainement, d'accélérer le rythme des cours. En plus de ces avantages, ces notes permettent d'accrô̂tre les éléments de jugement de l'étudiant, ainsi que sa participation aux traductions faites en classe et de diversifier le contenu des examens.

Sur le plan psychologique, l'étudiant se sent plus encadré étant donné que les notes sont propices à un dialogue, et donc à une meilleure compréhension, entre le professeur et l'étudiant.

En conclusion, c'est essentiellement à l'étudiant que les notes d'orientation peuvent être utiles, et c'est par voie de conséquence qu'elles le sont aussi pour le professeur. Elles doivent permettre au premier d'acquérir des réactions sûres dès le départ, et d'aiguiser son sens de la recherche, avant que ne prennent naissance chez lui des habitudes erronées de segmentation ou de transformation linguistiques. Elles pourront aussi prévenir par la suite bien des rectifications parfois brutales, de la part de réviseurs, une fois le traducteur lancé sur le marché du travail. 